\title{
Canine Extremity Wound Treatment with Chitosan Extracted from Shrimp Shells: A Case Report
}

\author{
Bilge Kaan Tekelioglu ${ }^{1}$, Mehmet Celik ${ }^{2}$ and Aygul Kucukgulmez ${ }^{3}$ \\ 1. Department of Virology, Faculty of Ceyhan Veterinary Medicine, Cukurova University, Adana 01930, Turkey \\ 2. Department of Food Hygiene and Technology, Faculty of Ceyhan Veterinary Medicine, Cukurova University, Adana 01930, \\ Turkey
}

3. Department of Fisheries and Fish Processing Technology, Fisheries Faculty, Cukurova University, Adana 01930, Turkey

\begin{abstract}
The aim of this case report was to demonstrate the effectivity of chitosan as topical agent on the treatment of severely infected canine extremity wound. The dog was hit by a bus and presented severe friction wounds accompanying with bilateral hip and extremity fractures. Local and generalized infections were observed on the post operational period. The dog was unresponsive to the standard therapeutic protocols and health status was getting worsened. The regenerative sutures did not hold the tissues because of the infection and the tissue eruption. Chitosan samples were extracted from shrimp shell wastes. The milled form of chitosan was topically used to treat the open and heavily mix infected wound areas located at skin and rear extremities accompanying with fever and Canine coronavirus diarrhea. It was decided to use the chitosan as a bio barrier and anti-bio affect over the damaged and erupted tissues. Macroscopic findings indicated chitosan promoted the natural blood clotting and absorbed the inflammation fluid. Microscopic findings indicated the infiltration of polymorph nuclear leukocytes (PMN) accelerated with the topical use of chitosan in the early phase of wound healing. The wound healing was observed daily. This veterinary practice wound treatment result indicated that chitosan is effective to medicate topically the canine open and mix infected wounds with bacteria and viruses and promotes the granulomatosis in four weeks period.
\end{abstract}

Key words: Chitosan, canine wound healing, shrimp shells.

\section{Introduction}

Canine wound abscesses are generally associated with infection, trauma, frictions, post-surgery, bites, puncture wounds or other dermal problems which affect the epidermis, dermis and subcutaneous layers either individually or together. Inflammations, infections and fever frequently follow by a thick yellowish color discharge called as pus. The abscesses may affect the nerves, cause pain, ruptures and immobility, and also would lead to life threatening situations if untreated properly. The major risk is spreading the infections to the tissues and organs of the body via blood circulation which called as sepsis. The wound healing and granulation may not occur or

Corresponding author: Bilge Kaan Tekelioglu, assistant professor, research fields: virology, microbiology and infectious diseases. be delayed with abscesses. Post traumatic hemorrhage remains one of the most mortal causes of early death with progressive seconder infections in wounds, although chitosan dressings were developed to address these problems, minimize scarring, strengths new tissue, provides protein for healing, absorbs fluids from inflammation, blocks nerve endings to reduce pain, encourages natural blood clothing, forms barrier against infection and provides scaffold for cell growth [1-9]. There are insufficient data in Turkey on canine wound and abscess treatment. Anecdotally the usage of broad band spectrum antibiotics and commercial wound sprays are common.

It has been reported that chitosan has strong antimicrobial effects against different groups of microorganism, such as bacteria, fungi and yeast, and was also found affective on wound healing [1-3]. 
Chitin is reported as the second most common polymer after cellulose in nature, existing in the shells of crustaceans, like shrimp [4-7]. Chitosan receives increasing attention as one of the promising renewable polymeric materials for various applications, such as wound healing and hemostatic activity [3, 4, 8, 9]. Cheung et al. reported [10] bio-molecules of marine origin with a defense action against pathogenic viruses and fungi. Usami et al. [11, 12] reported that the bovine and canine polymorph nuclear cells in vitro activation induced by chitin and chitosan [13]. The ability of chitin fragments to regulate murine macrophage cytokine in vitro production was described by Da silva et al. [14]. Okamoto et al. [15] evaluated the histological effects of chitin obtained from squid pen on dogs. Moreover, Okamoto et al. [16] described the sponge-cotton and flake-type remedies made of chitin applied to various types of trauma, abscess, surgical tissue defect and herniorrhaphy in 147 clinical cases including, 72 dogs. In this paper, a represented case of three years old female German shepherd dog was reported. The aim was to investigate the efficiency of chitosan topically used as a biocompatible material on both to promote wound healing and hemostatic activity.

\section{Materials and Methods}

In this study, a represented case was reported. A three years old female German shepherd dog was consulted in an emergency situation after a heavy traumatic traffic accident with severe wounds and fractures, which were visualized by $x$-ray examination. The dog was in coma, hypothermia and life threatening health status on the arrival to emergency room because of excessive blood lose and trauma. The wounded areas coverage was around $25 \%$ of the body. There were impact and friction wounds made by asphalt effect on both side of the rear legs and hip. The wounds were cleaned and washed with saline solution $(0.90 \% \mathrm{w} / \mathrm{v}$ of $\mathrm{NaCl})$ and treated with $3 \%$ hydrogen peroxide and $10 \%$ providone iodine poli-iode complex solution immediately. The dog was stabilized within the next $3 \mathrm{~d}$ under intensive care with intravenous (IV) fluid replacement and wound treatment associated with antimicrobial supports. Osteo synthesis and wound surgery was performed against hip, fibula and tibias shaft fractures. Ceftriaxone $30 \mathrm{mg} / \mathrm{kg}$ (IV) and 10\% providone iodine topical solution as antibacterial agent were used twice daily during the post-operative period. .

Smears and sterile swabs were used for sampling the wound discharge and stool. The $96 \%$ ethanol was used to fixation, Giemsa (Merck ${ }^{\mathrm{R}}$ ) and Gram protocol was used to stain. Stool serology to detect the Canine coronavirus antigen was done by a commercial kit (Fassisi Corona $^{\mathrm{R}}$, sensitivity 96.15\%, specifity 95.65\%). A commercial probiotic diet supplement against viral diarrhea (Diazyme $256^{\mathrm{R}}$ ) was used. Mc Conkey agar (MCA) and sheep blood agar (SBA) was used for bacterial cultures and hemolysis activity incubated at $37{ }^{\circ} \mathrm{C}$ for $24 \mathrm{~h}$. Gram stain was used; heat-fixed smear of the bacterial culture stained with crystal violet, followed by iodide stain to bind and traps the crystal violet in the cells. The $96 \%$ ethanol rapid decolorization was followed by carbol fuchsine stain. Seborrheic dextrose agar (SDA) and triptych soy broth (TSB) used for yeast culture. Müller Hinton (MH) agar and antibiotic impregnated absorbent papers in conformity with standards (Bioanalyse ${ }^{\mathrm{R}}$ ) was used for antibiotic susceptibility test (AST). Free coagulase test was done with freeze-dried rabbit plasma $\left(\right.$ Bio-Rad $^{\mathrm{R}}$ ). $3 \% \mathrm{H}_{2} \mathrm{O}_{2}$ was used for catalase test, Kovacs oxidase reagent (1\% tetra-methyl-p-phenylene diamine dihydrochloride (in water) used for direct plate oxidase test, and O-F glucose medium which consists of a $1 \%$ glucose added nutrient medium with oil overlay, used to test the O-F glucose activity.

Chitosan was extracted from shrimp shells according to the description by Kucukgulmez et al. [5, 6]. Deproteinization and demineralization steps were carried out with $2.5 \mathrm{~N} \mathrm{NaOH}$ at $65^{\circ} \mathrm{C}$ for $6 \mathrm{~h}$ and 1.7 
$\mathrm{N} \mathrm{HCl}$ at $25{ }^{\circ} \mathrm{C}$ for $6 \mathrm{~h}$, respectively. The chitin residue was treated with nine volumes of hydrogen peroxide (one volume chitin: nine volumes $\mathrm{H}_{2} \mathrm{O}_{2}$ ) and dried at $90{ }^{\circ} \mathrm{C}$ for 2 h. Deacetylated chitosan was prepared by alkali treatment of chitin using 50\% $\mathrm{NaOH}(\mathrm{w} / \mathrm{v})$ in distilled water at $120^{\circ} \mathrm{C}$. The reactants were filtered, washed with deionized water to neutral $\mathrm{pH}$ and dried at $90{ }^{\circ} \mathrm{C}$ for $2 \mathrm{~h}$ to get the chitosan powder (Fig. 1). Based on the data and results, chitosan represents antimicrobial activity $(++)$ against S. aureus. The antimicrobial activity of extracted chitosan is presented at Table 1, while physicochemical properties (moisture and saponification, degree of de-acetylation, molecular weight, water and fat binding capacities and apparent viscosity) was measured by Kucukgulmez et al. [5, 6] and presented at Table 2. Chitosan has a very high efficiency on water binding capacity of $712.99 \% \pm$ $11.98 \%$, and fat binding capacity of $531.15 \% \pm$ $12.26 \%$.

The dog did not response the standard therapeutic protocols and health status was getting worsened. The regenerative sutures did not hold the tissues because of the infection and the tissue eruption, so the decision has to be done either by an amputation of an extremity or to change wound healing protocols. Also it was indispensable to promote the natural blood clothing and cell growth with the effect of an inflammation fluid absorbent. The wounds were washed with saline solution $(0.90 \% \mathrm{w} / \mathrm{v}$ of $\mathrm{NaCl})$ first before the extracted milled chitosan was totally dusted topically once a day and covered with sterile cotton gauze and bandaged at first $7 \mathrm{~d}$. The same protocol was followed within the next $21 \mathrm{~d}$, except the wounded areas were not covered neither with the gauze or bandage to promote aeration and oxygenation of the tissues. The usage of antibiotic is also limited to balance the general good health status and control seconder infections. During the study by microscopy microbial and polymorph nuclear leukocytes (PMN) cells (segmented and toxic neutrophils, small lymphocytes) change and differentiation were followed. Re-epithelialization, granulomatosis, healing levels and other recuperation signs were followed by macro observations. This case report is based on the macro, micro and histopathological observations performed before and during the represented case of a naturally wounded canine which topically medicated by milled chitosan.

\section{Results and Discussion}

\subsection{Clinical Approach and Patient History}

Post traumatic clinical and X-ray examination indicated bilateral multiple structural fibula, tibia and pelvic fractures (Fig. 2). In the post-operative period, the supportive therapy, which included a broad spectrum antibiotic, IV fluids and diet, were administered to the

Table 1 Antimicrobial activity of extracted chitosan.

\begin{tabular}{|c|c|c|c|c|c|c|c|c|c|c|}
\hline \multirow{2}{*}{$\begin{array}{l}\text { Concenteration } \\
(\%)\end{array}$} & \multicolumn{10}{|c|}{ Diameter of inhibition zone of microorganisms (mm) } \\
\hline & Pp & Pf & Vp & Se & Sa & Ec1 & Ec2 & $\mathrm{Lm}$ & Ef & Ac \\
\hline 1.00 & ++ & ++ & ++ & ++ & + & ++ & ++ & + & ++ & + \\
\hline 0.50 & ++ & ++ & ++ & ++ & ++ & + & + & ++ & ++ & + \\
\hline 0.25 & ++ & ++ & ++ & ++ & ++ & - & ++ & +++ & ++ & + \\
\hline 0.10 & +++ & +++ & ++ & ++ & ++ & - & ++ & +++ & ++ & ++ \\
\hline 0.005 & ++ & ++++ & +++ & + & ++ & - & ++ & +++ & ++ & ++ \\
\hline
\end{tabular}

Pp: Pseudomonas putida NRRL-B-14875; Pf: Pseudomonas fluorescens NRRL-2641; Vp: Vibrio parahaemolyticus ATCC-17802; Se: Staphylococcus epidermidis NRRL-B-4268; Sa: Staphylococcus aureus ATCC-25923; Ec1: Escherichia coli ATCC-25922; Ec2: Escherichia coli NRRL-B-4269; Lm: Listeria monocytogenes ATCC-7644; Ef: Enterecoc faecalis ATCC-29212; Ac: Aeromonas caviae NRRL-968.

Results were interpreted in terms of the diameter of the inhibition zones: -: < 7.00 mm; +: 7.00-9.0 mm; ++: 9.0-11.0 mm; +++: 11.0-14.0 mm; ++++: 14.0-16.0 mm; +++++: > $16.00 \mathrm{~mm}$. 
Table 2 The physicochemical characterization of extracted chitosan by Kucukgulmez et al. [5].

\begin{tabular}{ll}
\hline Physicochemical parameters & Values $^{*}$ \\
\hline Moisture (\%) & $1.33 \pm 0.08$ \\
Ash (\%) & $0.61 \pm 0.03$ \\
Deacetylation degree (\%) & $92.19 \pm 2.56$ \\
Molecular weight (kDa) & $2.20 \pm 0.03$ \\
Apparent viscosity (cP) & $46.14 \pm 0.07$ \\
Water binding capacity (\%) & $712.99 \pm 11.98$ \\
Fat binding capacity (\%) & $531.15 \pm 12.26$ \\
\hline
\end{tabular}

${ }^{*}$ Mean \pm standard deviation of triplicate determinations.

patient. However, the dog did not recover, and both local and diffuse infections observed on the both side of the wounded and surgery areas at early stages (Figs. 3 and 4) associated with fever, appetite and weight loss, anemia, rehydration, muscle atrophy and diarrhea. In the presence of infections, supportive antibiotic and IV fluid replacement with diet change was done. Probiotics were used to control intestinal health against $C$. coronavirus diarrhea and prescript by the producer was only able to colonize at intestines and works like an intestinal bio-barrier. The probiotics dermis colonization was ineffective and there was just limited effect for total good health status; the usage was limited only to control the $C$. coronavirus diarrhea. Similarly, Okamoto et al. [16] used chitosan as wound dressing and reported good healing in 28 out of 32 cases (87.5\%). Hillier et al. [17] reported that one of the major problems is lack of in vivo studies that assess the clinical efficacy and safety of topical therapy, either alone or in combination with systemic antimicrobial drugs (AMD) therapy and the absence of susceptibility interpretative criteria for topical agents.

\subsection{The Clinical Response}

The in vivo observations of the study indicated that the topical usage of chitosan as antimicrobial therapy could promote recovery and enhance resistance to infection. Chitosan is outstanding as a significant option, as systemic therapy becomes more limited. It was reported that with the absent of abscesses, the drainage of the pus and local or topical medication promotes the effectiveness of antibiotics to the skin [17]. It has also been reported that dermal infections are resolved after three weeks or more of systemic AMD treatment. Total resolution of all lesions and prevention of rapid recurrence of disease requires 3-6 weeks of treatment [17].

In this study, chitosan was found effective to absorb fluids from inflammation (Fig. 5), also it promoted natural tissue regeneration (Fig. 6), formed barrier against infection and minimized scarring (Fig. 7), promoted regeneration of the new tissue (Fig. 8), remodelling, cell growth and healing (Fig. 9). The findings were supported by Azuma et al. [4], who reported that chitosan promoted wound healing, Paul and Sharma [7] reported the haemostatic and re-ephitelization properties of chitosan at wound healing, Jayakumar et al. [9] reported that chitosan formed barrier against infection and Usami et al. [12] reported that wound healing was accelerated with the presence of chitosan by inducing the chemotaxis of nutrophils. The inflammatory cell infiltration with a remarkable accumulation of PMN was observed in the study, which was clearly reported by Usami et al. [11-13] and Da Silva et al. [14].

\subsection{Microscopic Findings}

At the microscopy, segmented and toxic neutrophils, small lymphocytes, and the round shaped bacteria were observed (Figs. 10 and 11). Bacterial growth with alpha-hemolysis was observed on SBA incubations at $37^{\circ} \mathrm{C}$ for $24 \mathrm{~h}$, but not on MCA. No pathogenic yeast cell was observed at TSB and SDA. Gram stain results indicated bunches of grapes formation which is typical to Staphylococcus spp. (Fig. 12). Fresh colonies used to perform the AST. MH agar was used for the test with the inoculum of the bacteria at $37{ }^{\circ} \mathrm{C}$ for $24 \mathrm{~h}$. The ciprofloxacin susceptible Staphylococcus spp. was isolated. Stool sample was tested with the serological C. coronavirus antigen test and seropositivity was observed. Isolated bacteria 
were inoculated with $0.5 \mathrm{~mL}$ rabbit plasma in tube overnight at $37^{\circ} \mathrm{C}$ to test the coagulase activity. The positive result of the clotting of the plasma was observed. To test the catalase activity, $3 \% \mathrm{H}_{2} \mathrm{O}_{2}$ drop mixed on a slide with fresh inoculated bacteria sample and bubbles was observed as positive result. An inoculum is transferred aseptically to a tube of O-F glucose medium with oil overlay. The inoculated tube is incubated at $37^{\circ} \mathrm{C}$ for $24 \mathrm{~h}$ and the fermentation of glucose with the color change to yellow was observed. Results indicated that the dog was infected with the $S$. aureus and C. coronavirus. It was observed well effective the additional probiotics to the diet against viral diarrhea, IV fluid support and topical use of extracted chitosan powder together with the change of antibiotic (ciprofloxacin $15 \mathrm{mg} / \mathrm{kg}$ by mouth twice daily). The dog was stabilized back again and recovered. At the microscopic evaluation on day 0 , PMN cells under the oil immersion observation area as identified as small lymphocytes, segmented neutrophils and cocci bacteria, were observed (Fig. 10). At day 7, through the direct microscopic observations of giemsa stained smears with oil immersion, the segmented neutrophils, toxic neutrophils and round bacteria were observed, indicating increasing numbers of PMN cells in the absence of macrophages and granulomatous inflammation (Fig. 11).

\subsection{Recovery}

The high physicochemical properties on water binding capacity indicate that chitosan is a very efficient adsorbent and safe to use on wound healing protocols (Fig. 5). The usage of antibiotic is also limited to balance the general good health status and control seconder infections. The findings indicated topical chitosan was very obvious, measurable and quick-acted. The use of topical agents at canine deep pyoderma was known as one of the golden standard.

Up to the findings, it was shown that the application of chitosan significantly delayed the appearance of macrophages. The clinical, macro and microscopic results indicated that chitosan materials promoted healing tissue regeneration and without any side effect. The microscopic histological evidences of the data indicated that the induced inflammatory cell migration and angiogenic activity promoted a high vascularization of the regenerative tissue (Figs. 6 and 7). The findings were overlapped by Balazzi et al. [1], Azuma et al. [4], Usami et al al. [12], Da silva et al. [14], Alemdaroglu et al. [18] and Sezer et al. [19]. Post traumatic hemorrhage remains one of the most mortal causes of early death with progressive seconder infections in wounds. Results indicated that chitosan is a good antimicrobial barrier as reported before [9, 20-23]. The dog were stabilized and immobilized for eight weeks. Immobility caused pressure negatively affected both the moral of the animal and the wound healing levels of recovery. The efficacy of chitosan in closing pressure ulcers was also reported as a potential therapeutic agent by Park [24]. Additionally, it has been observed the chitosan's chemotactic activity promotes neutrophils without enforcing any antimicrobial activity directly to chitosan. This phenomenon may explain the chitosan as a biodegradable and bio-origin material which promotes the coagulation, early and late inflammation, proliferation and finally remodeling without any inflammatory response directly to itself $[4,8,12-15$, 24]. Finally, under the results of the case report, chitosan based wound materials were found to be good and effective in wound treatment and infection

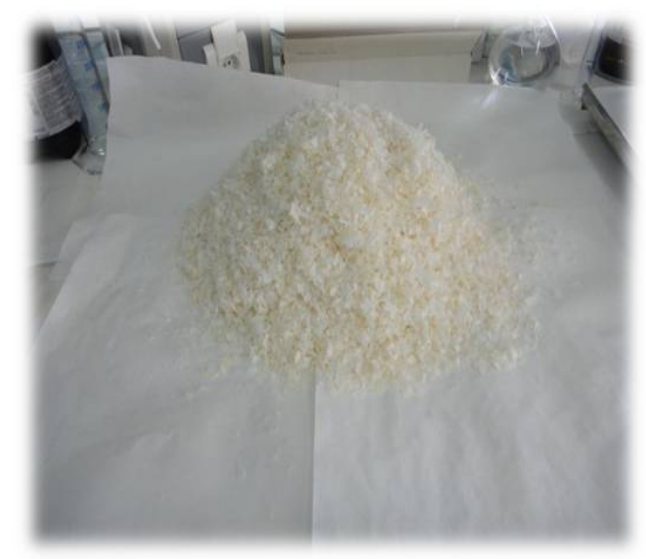

Fig. 1 In-lab extracted milled chitosan. 


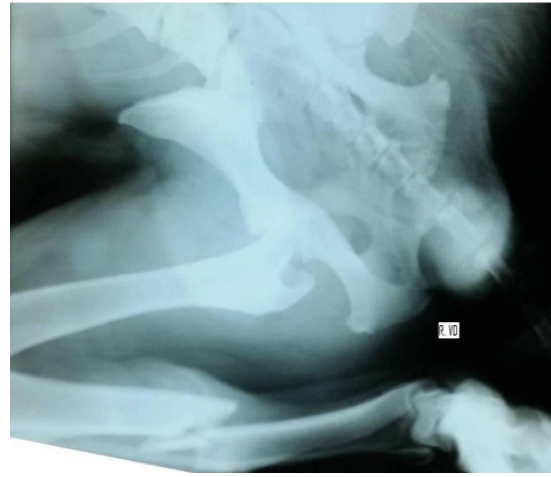

Fig. 2 Post traumatic X-ray image.

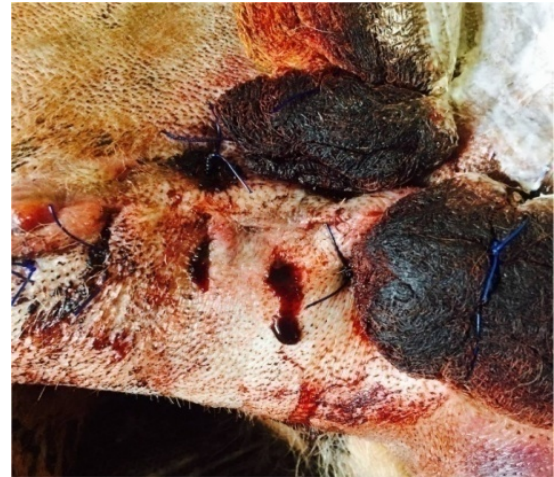

Fig. 3 Infected wound, subcutaneous abscess and discharges of the reported canine at post surgery and before chitosan period.

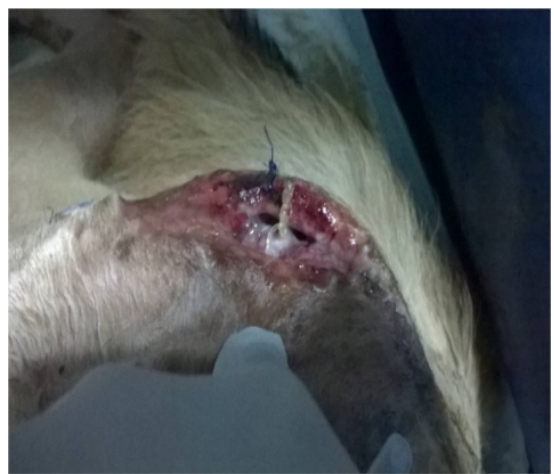

Fig. 4 Infected discharge and erupted sutures.

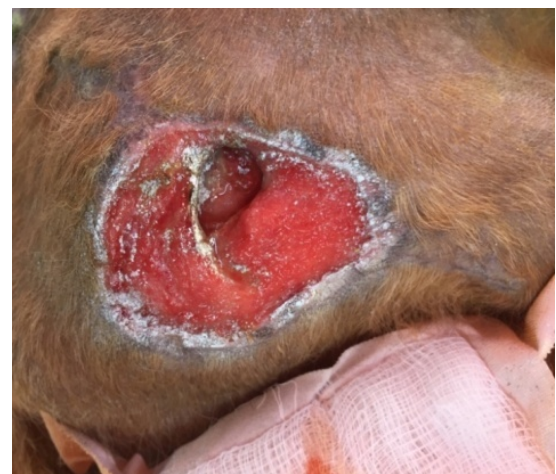

Fig. 5 Adsorbent activity at day 0 after chitosan treatment.

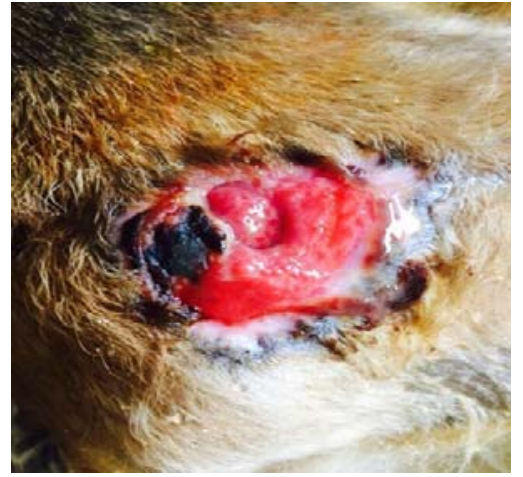

Fig. 6 Regeneration and proliferation at day 7 after chitosan treatment.

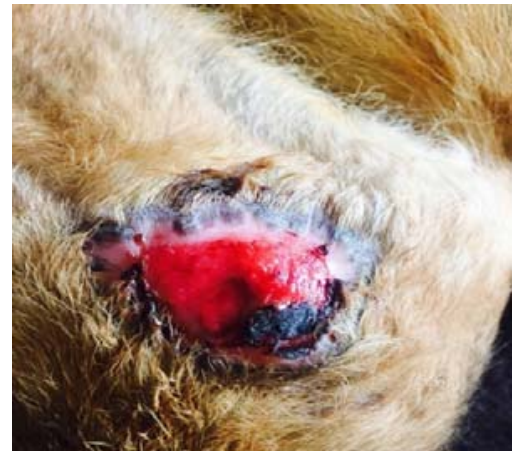

Fig. 7 Formed barrier against infection and minimized scarring at day 14 after chitosan treatment.

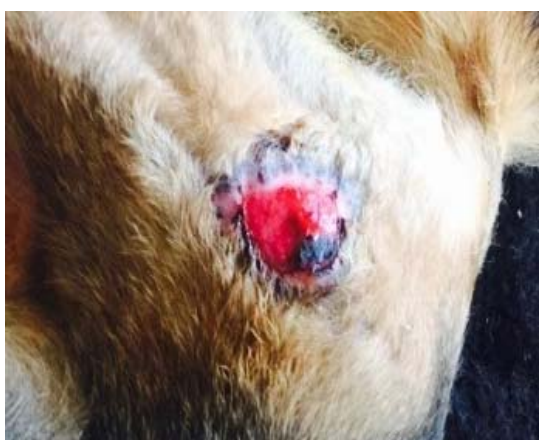

Fig. 8 Promoting regeneration of the new tissue at day 21 after chitosan treatment.

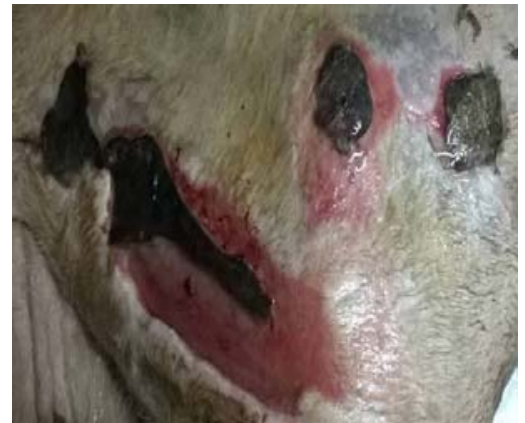

Fig. 9 Remodelling, cell growth and healing at day 28 after chitosan treatment. 


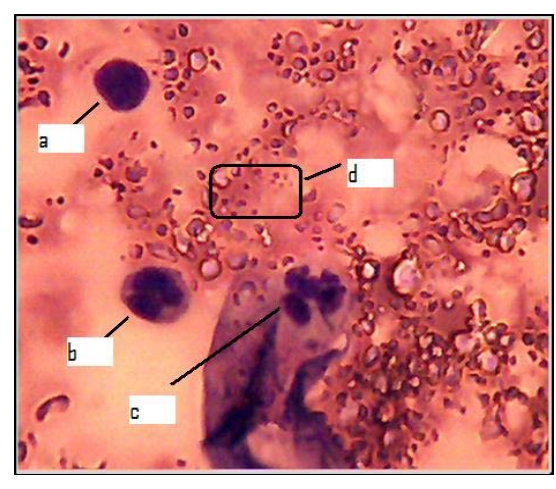

Fig. 10 Gimza stained microphotograph at day 0.

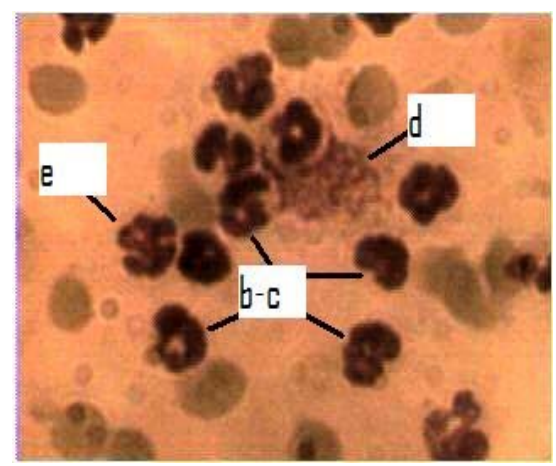

Fig. 11 Gimza stained microphotograph at day 7.

Arrow a: small lymphocytes; b and c: segmented neutrophils; d: round shape bacteria; e: toxic neutrophils.

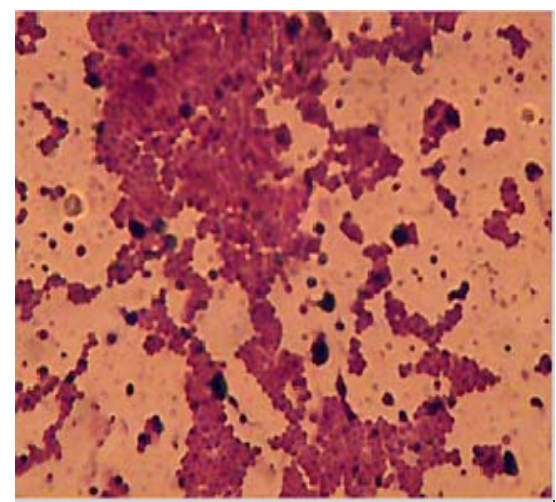

Fig. 12 Isolated Staphylococcus aureus from the wound.

control in veterinary medicine, as reported earlier by Okamoto et al. [15, 16].

\section{Conclusions}

This case study reported the observations on the effect of chitosan on animal wound treatment and antimicrobial activity. The chitosan was previously reported to be effective in promoting wound healing and to control infections, so this paper was to report the results of clinical treatment in a very complicated case. In vitro studies may not be overlapped at all times with in vivo responses, thus field trials are important to implement the alternative non-antibiotic antimicrobials. From the beginning to the end, every immediate effort was done to survive the patient, including bacteriology, dietetic and nutrition, immediate life support, intensive care, mycology and virology. The dog has open, infected fistulas and erupted wounds which were unresponsive to routine therapy. Topical use of chitosan affected well and absorbed the discharge. The clinical, macro and microscopic results indicated that chitosan materials promoted healing tissue regeneration and without any side effect. The histological evidences indicated that the induced inflammatory cell migration and angiogenic activity promoted a high vascularization of the regenerative tissue. It can be observed that chitosan is a good antimicrobial barrier and effective to absorb fluids from inflammation, encourage natural blood clotting, minimize scarring, strength new tissue, form barrier against infection and promote regeneration and cell growth.

\section{References}

[1] Balassa, L. L., and Prudden, J. F. 1984. “Applications of Chitin and Chitosan in Wound Healing Acceleration.” In Chitin, Chitosan and Related Enzymes, edited by Zikakis, J. P. San Diego, California: Academic Press, 296-305.

[2] Muzzarelli, R. A. A. 1993. "Biochemical Significance of Exogenous Chitins and Chitosans in Animals and Patients." Carbohydrate Polymers 20: 7-16.

[3] Bottomley, K. M. K., Bradshaw, D., and Nixon, J. S. 1999. Metalloproteinases as Targets for Anti-inflammatory Drugs. Basel: Birkhauser.

[4] Azuma, K., Izumi, R., Osaki, T., Ifuku, S., Morimoto, M., Saimoto, H., Minami, S., and Okamoto, Y. 2015. “Chitin, Chitosan and Its Derivatives for Wound Healing: Old and New Materials.” J. Funct. Biomater. 6 (1): 104-42.

[5] Küçükgülmez, A., Celik, M., Yanar, Y., Sen, D., Polat, H., and Kadak, A. E. 2011. "Physicochemical Characterization of Chitosan Extracted from Metapenaeus stebbingi Shells.” Food Chem. 126: 1144-8.

[6] Küçükgülmez, A., Gülnaz, O., Celik, M., Yanar, Y., Kadak, A. E., and Gerçek, G. 2012. “Antimicrobial Activity of the Chitosan Extracted from Metapenaeus stebbingi Shell Wastes.” J. Polym. Environ. 20 (2): 
431-7.

[7] Paul, W., and Sharma, C. P. 2004. "Chitin and Alginates Wound Dressings: A Short Review.” Trends Biomater. Artif. Organs 18 (1): 18-23.

[8] Li, H. L., Deng, J. C., Deng, H. R., Liu, Z. L., and Li, X. L. 2010. "Preparation, Characterization and Antimicrobial Activities of Chitosan/Ag/ZnO Blend Films.” Chem. Eng. J. 160: 378-82.

[9] Jayakumar, R., Prabaharan, M., Sudheesh, K. P. T., Nair, S. V., and Tamura, H. 2011. "Biomaterials Based on Chitin and Chitosan in Wound Dressing Applications.” Biotechnology Advances 29 (3): 322-37.

[10] Cheung, R. C., Wong, J. H., Pan, W. L., Chan, Y. S., Yin, C. M., Dan, X. L., Wang, H. X., Fang, E. F., Lam, S. K., Ngai, P. H., Xia, L. X., Liu, F., Ye, X. Y., Zhang, G. Q., Liu, Q. H., Sha, O., Lin, P., Ki, C., Bekhit, A. A., Bekhit, A. E. D., Wan, D. C., Xia, J., and Bun, T. N. 2014. “Antifungal and Antiviral Products of Marine Organisms." Appl. Microbiol. Biotechnol. 98 (8): 3475-94.

[11] Usami, Y., Okamoto, Y., Minami, S., Matsuhashi, A., Kumazawa, N. H., Tanioka, S., and Shigemasa, Y. 1994. "Chitin and Chitosan Induce Migration of Bovine Polymorphonuclear Cells.” J. Vet. Med. Sci. 56 (4): 761-2.

[12] Usami, Y., Okamoto, Y., Minami, S., Matsuhashi, A., Kumazawa, N. H., Tanioka, S., and Shigemasa, Y. 1994. "Migration of Canine Neutrophils to Chitin and Chitosan." J. Vet. Med. Sci. 56 (6): 1215-6.

[13] Usami, Y., Minami, S., Okamoto, Y., Matsuhashi, A., and Shigemasa, Y. 1997. "Influence of Chain Length of N-Acetyl-D-Glucosamine and D-Glucosamine Residues on Direct and Complement-Mediated Chemotactic Activities for Canine Polymorphonuclear Cells.” Carbohydrate Polymers 32: 115-22.

[14] Da Silva, C. A., Hartl, D., Liu, W., Lee, C. G., and Elias, J. A. 2008. "TLR-2 and IL-17A in Chitin-Induced Macrophage Activation and Acute Inflammation.” $J$. Immunol. 181 (6): 4279-86.

[15] Okamoto, Y., Minami, S., Matsuhashi, A., Sashiwa, H., Saimoto, H., Shigemasa, Y., Tanigawa, T., Tanaka, Y., and Tokura, S. $1993 . \quad$ "Polymeric N-Acetyl-D-glucosamine (Chitin) Induces Histionic Activation in Dogs.” J. Vet. Med. Sci. 55 (5): 739-42.

[16] Okamoto, Y., Minami, S., Matsuhashi, A., Sashiwa, H.,
Saimoto, H., Shigemasa, Y., Tanigawa, T., Tanaka, Y., and Tokura, S. 1993. "Application of Polymeric N-Acetyl-D-Glucosamine (Chitin) to Veterinary Practice.” J. Vet. Med. Sci. 55 (5): 743-7.

[17] Hillier, A., Lloyd, D. H., Weese, J. S., Blondeau, J. M., Boothe, D., Breitschwerdt, E., Guardabassi, L., Papich, M. G., Rankin, S., Turnidge, J. D., and Sykes, J. E. 2014. “Guidelines for the Diagnosis and Antimicrobial Therapy of Canine Superficial Bacterial Folliculitis (Antimicrobial Guidelines Working Group of the International Society for Companion Animal Infectious Diseases)." Vet Dermatol. 25 (3): 163-75.

[18] Alemdaroglu, C., Degim, Z., Celebi, N., Zor, F., Ozturk, S., and Erdogan, D. 2006. "An Investigation on Burn Wound Healing in Rats with Chitosan Gel Formulation Containing Epidermal Growth Factor.” Burns. 32 (3): 319-27.

[19] Sezer, A. D., Hatipoglu, F., Cevher, E., Ogurtan, Z., Bas, A. L., and Akbuga, J. 2007. "Chitosan Film Containing Fucoidan as a Wound Dressing for Dermal Burn Healing: Preparation and in Vitro/in Vivo Evaluation." AAPS Pharm. Sci. Tech. 8 (2): 94-101.

[20] Dai, T., Tanaka, M., Huang, Y. Y., and Hamblin, M. R. 2011. "Chitosan Preparations for Wounds and Burns: Antimicrobial and Wound-Healing Effects.” Exp. Rev. Anti Infect. Ther. 9 (7): 857-79.

[21] Rabea, E. I., Badawy, M. E. T., Stevens, C. V., Smagghe, G., and Steurbaut, W. 2003. "Chitosan as Antimicrobial Agent: Applications and Mode of Action.” Biomacromolecules 4 (6): 1457-65.

[22] Li, P., Poon, Y. F., Li, W. F., Zhu, H. Y., Yeap, S. H., Cao, Y., Qi, X. B., Zhou, C. C., Lamrani, M., Beuerman, R. W., Kang, E., Mu, Y. G., Li, C. M., Chang, M. W., Leong, S. S. J., and Chan-Park, M. B. 2011. “A Polycationic Antimicrobial and Biocompatible Hydrogel with Microbe Membrane Suctioning Ability.” Nature Mater. 10: 149-56.

[23] Kong, M., Chen, X. G., Xing, K., and Park, H. J. 2010. "Antimicrobial Properties of Chitosan and Mode of Action: A State of the Art Review." Int. J. Food Microbiol. 144 (1): 51-63.

[24] Park, C. J. 2008. "The Use of Chitosan for Treatment of Pressure Ulcers.” Ph.D. thesis, University of Illinois at Urbana-Champaign. 